\title{
Bots Sociais: Como robôs podem se tornar pessoas influentes no Twitter?
}

\author{
Johnnatan Messias $^{1}$, Fabrício Benevenuto ${ }^{2}$, Ricardo Oliveira ${ }^{1}$ \\ ${ }^{1}$ Departamento de Computação - Universidade Federal de Ouro Preto, UFOP, Brasil
}

${ }^{2}$ Departamento de Ciência da Computação - Universidade Federal de Minas Gerais, UFMG, Brasil

\{johnnatan20@gmail.com, fabricio@dcc.ufmg.br, rrabelo@gmail.com\}

\begin{abstract}
Systems that classify influential users in social networks has been used with great frequency, being referenced in scientific papers and the media as the ideal standard for evaluation of influence in the social network Twitter. We consider this measure a complex and subjective and therefore suspect vulnerability and ease of handling these systems. Based on this, we performed experiments and analyzes in two ranking systems of influence: Klout and Twitalyzer. We create simple robots capable of interacting through Twitter accounts and measure their influence. Our results show that it is possible to be influential through simple strategies. This suggests that the systems do not have ideal metric to rank influence.
\end{abstract}

Resumo. Sistemas que classificam usuários influentes nas redes sociais têm sido usados com grande frequência, sendo referenciados em artigos científicos e na mídia como padrão ideal para avaliação de influência na rede social Twitter. Consideramos esta medição algo complexo e subjetivo e por isso suspeitamos da vulnerabilidade e facilidade de manipulação nesses sistemas. Baseado nisto, realizamos experimentos e análises em dois sistemas de classificação de influência: Klout e Twitalyzer. Criamos robôs simples capazes de interagir, através de contas no Twitter e medimos suas influências. Nossos resultados mostram que é possível ser influente através de estratégias simples. Isso sugere que os sistemas não possuem métricas ideais para classificar influência.

\section{Introdução}

As redes sociais têm adquirido bastante destaque e crescente importância na sociedade moderna. Dentre elas, o Twitter tem conquistado grande quantidade de adeptos. É uma das redes sociais que mais movimenta usuários e uma das que mais geram troca de informações. Em sistemas como o Twitter, usuários podem influenciar e serem influenciados por outros, o que tem atraído grande interesse político e de empresas relacionadas ao marketing.

Neste contexto, várias empresas tem se especializado em medir influência no Twitter e em outras redes sociais. Dentre os sistemas mais populares estão o Klout[1] e o Twitalyzer[2], que utilizam abordagens de medições de influência simples e cujos detalhes não são revelados ao público.

Surpreendentemente, vários jornais, revistas e até mesmo artigos científicos [3], [4] tem utilizado essas ferramentas, e divulgado rankings de usuários influentes. Como 
exemplo, uma matéria do jornal The New York Times apresentou um estudo das pessoas mais influentes do mundo, baseado no Twitalyzer [5]. Entre os mais influentes, o estudo indicou o humorista brasileiro Rafinha Bastos, o rapper americano Snoop Dogg, o presidente dos Estados Unidos Barack Obama e o apresentador brasileiro Luciano Huck.

Baseado nisso, queremos investigar que estratégias podem tornar usuários comuns em usuários influentes, segundo rankings do Klout e Twitalyzer. Criamos algoritmos robôs simples capazes de interagir, através de contas no Twitter, como se fossem usuários na rede, trocando informações, seguindo e conquistando novos seguidores, durante um período de 90 dias. Os resultados das análises nos mostraram que as ferramentas Klout e Twitalyzer não apresentaram métricas ideais para classificar a influência de um indivíduo na rede. Constatamos que spammers espalhados na rede podem facilmente se passar por celebridades e pessoas populares, com um alto nível de influência baseado no Klout Score e Twitalyzer Impact.

Este trabalho foi realizado pelo aluno Johnnatan Messias, estudante de Ciência da Computação na Universidade Federal de Ouro Preto e responsável por todas as etapas de sua execução. O artigo é fruto de um projeto da disciplina Análise de Mídias Sociais ministrada pelo Prof. Fabrício Benevenuto(orientador) e também contou com a colaboração do Prof. Ricardo Oliveira(co-orientador).

\section{Trabalhos relacionados}

Alguns estudos apontam que um número enorme de seguidores de um usuário no Twitter é suficiente para determinar o seu grau de relevância. Outros estudos indicam que a influência de uma pessoa no Twitter está muito mais ligada ao conteúdo que ela compartilha do que com o número de pessoas que a seguem. Com isso, houveram várias tentativas e métodos para medir, de forma adequada, a influência dos usuários no Twitter [6], [7]. No trabalho de [8] é proposto o TwitterRank, um algoritmo baseado no PageRank, que usa tanto o grafo de conexões do Twitter quanto informações de tweets publicados para identificar usuários influentes. Por outro lado [9] usou agrupamento e classificação de mais de 15 características extraídas do grafo do Twitter e dos tweets postados por usuários para identificar os mais influentes.

No artigo de [10] foi investigado a atividade de link farms no Twitter e proposto formas para dissuadir tal atividade. Os autores constataram que um pequeno grupo de usuários legítmos, populares e ativos no Twitter são responsáveis pela maioria dos link farms. Eles buscam acumular capital social seguindo diversos usuários e conquistando novos seguidores. Com isso, spammers exploram este tipo de comportamento para conquistar seguidores e reputação no Twitter. Como forma de minimizar a influência deste tipo de comportamento, os autores propuseram um esquema de classificação, conhecido como Collusionrank, onde os usuários são penalizados por seguir spammers, reduzindo a influência de spammers e seus seguidores.

No trabalho de [11] foi verificado como as $O S N^{\prime} s$ são vulneráveis devido a infiltração em larga escala pelos socialbots: programas de computador capazes de controlar contas nas redes sociais e imitar usuários reais. Para isso, eles adotaram uma tradicional botnet baseada na web e construíram uma Socialbot Network, ou seja, um grupo de socialbots adaptativos. Foram coletados dados dos comportamentos dos usuários do Facebook, utilizando os socialbots. Acreditam, ainda, que infiltração em larga escala nas 
$O S N$ 's é somente uma das muitas outras ameaças na rede, sendo, portanto, necessário uma manutenção e melhoria na segurança das redes sociais para que os usuários fiquem mais seguros.

\section{Construção dos bots}

Antes de explicar o funcionamento dos bots, primeiramente vamos apresentá-los. Foram criados dois algoritmos robôs, usando a API Python do Twitter. O primeiro bot, denominado fepessoinha (https://twitter.com/fepessoinhas2) apenas segue automaticamente outros usuários e conquista novos seguidores. Para seguir os usuários, utilizamos o método de busca em largura, ou seja, a partir de um usuário inicial aleatório o algoritmo o inclui em uma lista, seguindo-o e selecionando aleatoriamente 30 usuários de sua lista de seguidos, que também são incluídos na lista. Feito isso, o processo será repetido de modo a seguir o próximo usuário, até o bot alcançar o limite de 2000 usuários seguidos, que é um limite imposto pelo Twitter. A Figura 1 mostra uma foto do perfil do bot fepessoinha ${ }^{1}$.

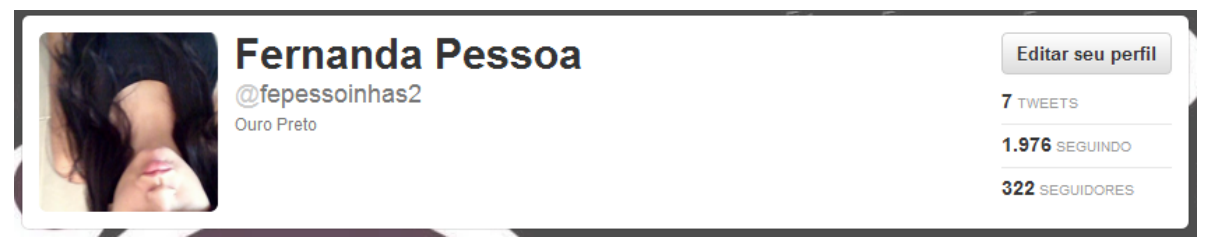

Figura 1. Perfil bot fepessoinha - Twitter

O segundo, denominado scarina (https://twitter.com/scarina91), é capaz de interagir, como se fosse um usuário na rede, trocando informações, seguindo e conquistando novos seguidores. Para seguir os usuários, este bot utiliza do mesmo princípio utilizado pelo bot fepessoinha, também conquistando consequentemente novos seguidores. Porém, este possui duas diferenças: (1)após seguir os 2000 usuários o algoritmo exclui os usuários que não seguem o bot e (2)é capaz de postar tweets. Para isso, uma sequência do algoritmo lê um dicionário de palavras relacionadas ao tema "Rede Globo". Colocamos no dicionário as seguintes palavras: rede, programação, xuxa, novela, filme, audiência, melhor, reportagem, jornalismo, jornal, nacional, huck, angélica, willian, bonner, bernardes, malhação, notícia, destaques, bbb. Assim, o algoritmo prossegue solicitando uma busca no Twitter através do trecho "Globo + palavra", sendo a palavra uma das selecionadas aleatoriamente no dicionário. Em seguida, capta quatro dos tweets retornados na pesquisa e os posta em um intervalo de tempo aleatório. Conforme as postagens aumentam, as atividades e interações entre os usuários com o bot scarina também aumentam. A Figura 2 mostra uma foto do perfil do bot scarina ${ }^{1}$.

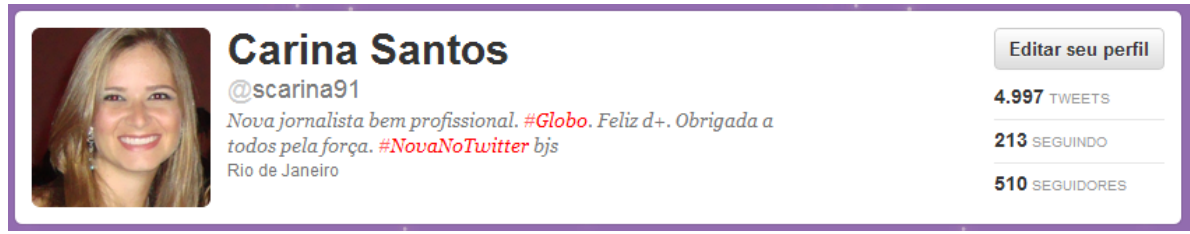

Figura 2. Perfil bot scarina - Twitter

${ }^{1}$ As fotos utilizadas no perfil de ambos os bots são fotos de conhecidas dos autores e que autorizaram o uso de suas fotos nos bots. 
Para conseguir automatizar os bots, de forma a respeitar as regras impostas pela API do Twitter. Os bots respeitam as seguintes restrições:

- Número máximo de requisições por hora = 350;

- Cada usuário pode seguir no máximo 2000 usuários, via API;

- Um usuário não pode seguir mais de 1000 usuários por dia.

\section{Experimentos com os bots}

Para analisar como os bots criados adquirem influência segundo o Klout e Twitalyzer, foram criados quatro cenários experimentais de forma a testar a vulnerabilidade e facilidade de manipulação dos classificadores de influência.

\subsection{Cenário 1: Seguindo usuários (Bot fepessoinha)}

O bot fepessoinha seguiu o limite máximo de usuários permitido pelo Twitter (2000), via API, na tentativa de obter seguidores.

\subsection{Cenário 2: Seguindo e excluindo usuários (Bot scarina)}

O bot scarina também seguiu o limite máximo de usuários permitido pelo Twitter (2000), via API. Posteriormente, o algoritmo do bot exclui alguns usuários, mantendo apenas os que também a seguiram. Neste cenário, o método utilizado para seguir usuários é o mesmo apresentado no cenário 1 , porém exclui posteriormente os usuários que não seguem o bot.

\subsection{Cenário 3: Seguindo, excluindo usuários e postando tweets (Bot scarina)}

O bot scarina posta tweets de forma automática, após realizar a estratégia do Cenário 2. Tal cenário permite verificar o impacto nos rankings de influência devido à postagem de conteúdo. Para isso utilizou um dicionário de dados para postagens de temas relevantes. Conforme as postagens aumentam, as atividades e interações entre os usuários com o bot scarina também aumentam.

\subsection{Cenário 4: Seguindo, excluindo usuários, postando tweets e com interrupções na execução (Bot scarina)}

O bot scarina segue e ganha novos seguidores além de postar tweets utilizando também o dicionário de dados. Neste cenário também verificamos e analisamos algumas interrupções e reinícios na execução do algoritmo do bot. Com o intuito de analisar o que acontece com a influência do bot quando este fica períodos de tempo inativo.

\section{Coleta de dados}

Para coletar os dados pertinentes para este artigo, na criação dos bots scarina e fepessoinha configuramos suas contas do Twitter a fim de enviar todas as informações aos e-mails cadastrados. Assim, teríamos um $\log$ de todas as ações, informações e atividades realizadas durante o período de 90 dias de experimento. Posteriormente, coletamos os e-mails, em arquivos ".eml", do servidor do Yahoo (serviço de $e$-mail das contas) e fizemos um algoritmo parser para filtrar as informações desejadas. Com isso, durante os 90 dias, conseguimos obter as seguintes informações do Twitter: tweets favoritos, tweets mencionados, mensagens, tweets respondidos, retweets e usuários seguidores. 
Após o processo de execução, acessamos os sites do Klout e Twitalyzer para coletar os resultados de todas as interações. No site da Klout foi possível coletar os resultados dos 90 dias de experimento, através de gráficos. Já no site da Twitalyzer, apenas conseguimos um resultado da influência final para cada usuário, desde o dia de sua criação. Não conseguimos dados dos dias anteriores ao de consulta.

Os dados coletados através dos e-mails servem para complementar os resultados obtidos pelos sistemas Klout e Twitalyzer e com isso achamos conveniente mostrar os resultados finais, para comprovar a vulnerabilidade e fácil manipulação dos sistemas Klout e Twitalyzer.

\section{Resultados}

Para se chegar ao Klout Score, o Klout trabalha com mais de 25 variáveis divididas em 3 grupos: Network Impact (Influência na rede), Amplification Probablity (Probabilidade de amplificação) e True Reach (Alcance Verdadeiro). O Klout Score vai de 0 à 100, e, teoricamente, é o resultado final do grau de influência de um usuário. No caso do Twitalyzer, este possui o número conhecido como Twitalyzer Impact. Este número também vai de 0 à 100 e baseia-se em 15 variáveis para se chegar ao valor final.

\subsection{Cenário 1}

O gráfico correspondente à Figura 3 mostra detalhadamente a quantidade de seguidores que o bot fepessoinha conquistou, resultando ao final do experimento o total de 417 seguidores. A quantidade de seguidores foi mais alta no momento em que o bot fepessoinha executou o processo de seguir os 2000 usuários (durante os primeiros 9 dias).

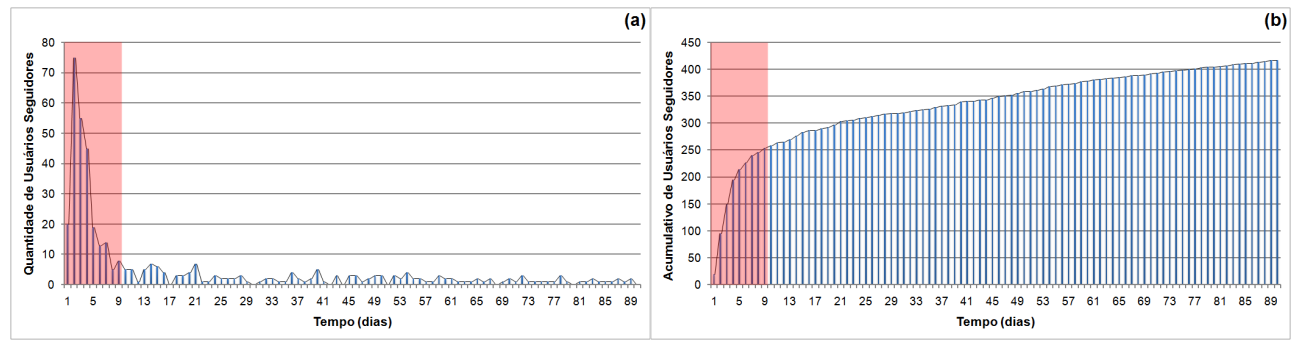

Figura 3. (a) Gráfico de Seguidores do bot fepessoinha (b) Gráfico do acumulativo de seguidores do bot fepessoinha

Com isso, podemos ver no gráfico correspondente à Figura 4, o resultado do Klout Score do bot fepessoinha. O Klout Score mostra o resultado final da influência de um usuário. É possível ver que nos 2 primeiros dias há uma grande crescente, devido aos novos usuários que passaram a seguir o bot, alcançando o valor 18 de Klout Score. Ao fim da execução do algoritmo do bot (após 9 dias) é possível ver uma queda constante do Klout Score até alcançar o valor 12,3 ao fim dos 90 dias de experimento.

Já no Twitalyzer, o bot conseguiu o valor 9, durante os 90 dias de experimento. Além desses resultados, o bot fepessoinha obteve durante os 90 dias de experimento: 0 tweets favoritados e 0 retweets, devido ao fato de ter publicado apenas 7 tweets. Desses 7, apenas 4 tweets foram respondidos. Conforme é mostrado na Figura 5 o bot obteve um valor considerável de tweets mencionados e mensagens recebidas resultando ao fim 


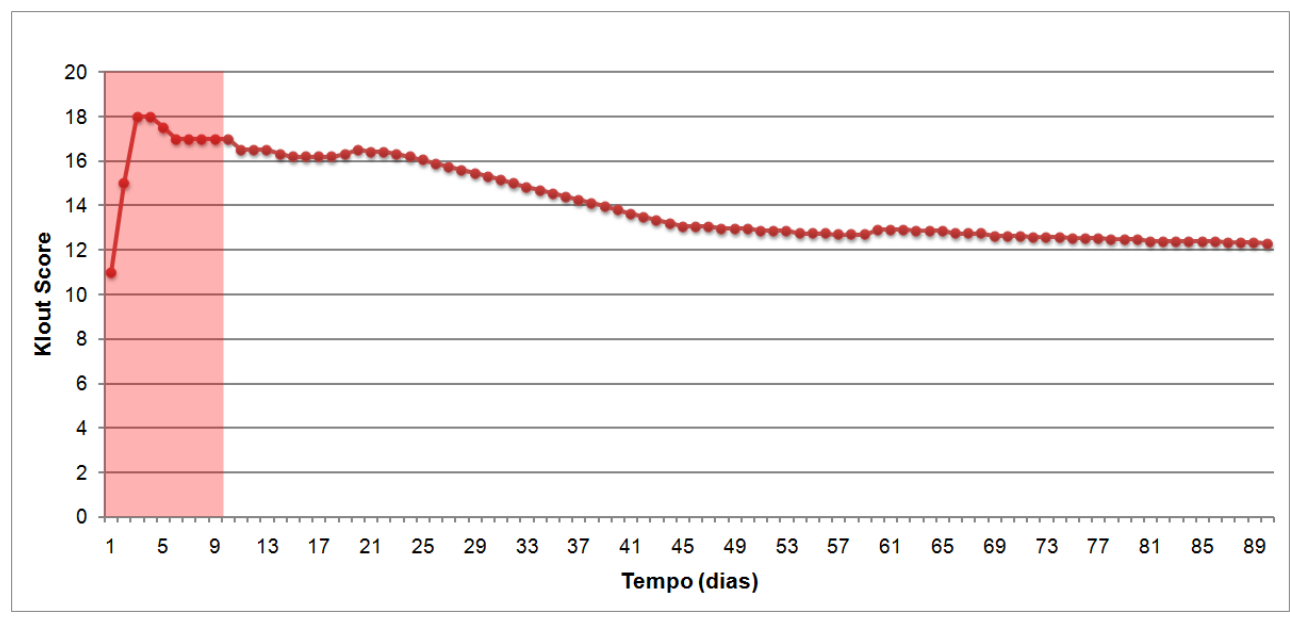

Figura 4. Resultados Klout Score do bot fepessoinha

do experimento o total de 24 e 21 respectivamente. Observamos que grande parte das menções e mensagens foram feitas nos 9 primeiros dias, nos dias em que o algoritmo de coleta de usuários do bot estava em execução. A maioria das menções e mensagens foram de questionamentos sobre quem era o bot fepessoinha, e de onde os usuários se conheciam. Um questionamento comum entre usuários que iniciam um relacionamento em uma rede social.
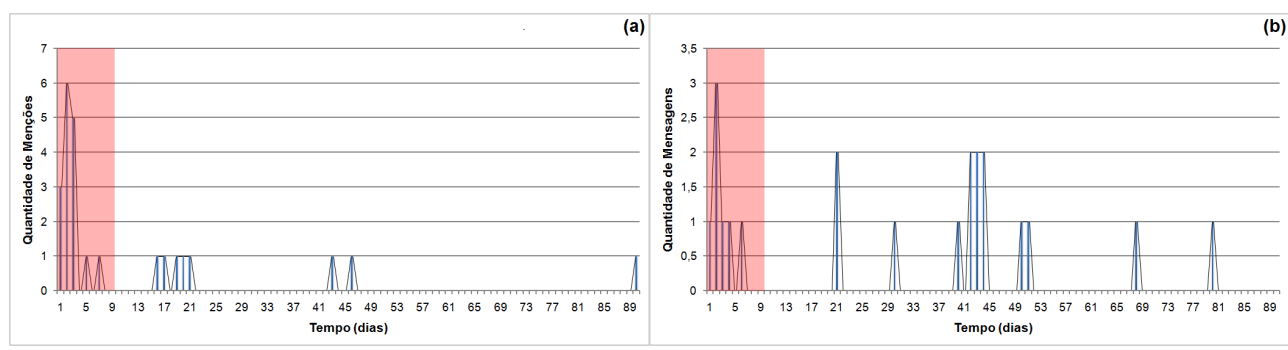

Figura 5. (a) Gráfico de Tweets mencionados do bot fepessoinha (b) Gráfico de mensagens do bot fepessoinha

\subsection{Cenário 2}

O gráfico correspondente à Figura 6 é o que expressa melhor o resultado deste experimento. Fazendo uma comparação com o bot fepessoinha, o bot scarina alcançou valores menores de influência. É possível ver que o fato do algoritmo do bot scarina excluir os usuários que não o seguem, sua influência acaba sendo menor do que o bot fepessoinha, que não exclui usuários, durante os 9 dias em que o algoritmo de coleta e exclusão de usuários foi executado. Após os 9 dias, ao fim do primeiro processo de coleta e exclusão de usuários, é iniciado o processo de postagem de tweets, iniciando o processo do Cenário 3.

\subsection{Cenário 3}

A partir do $10^{\circ}$ dia, apresentado pelo gráfico correspondente à Figura 6, podemos ver o início de uma discrepância brusca entre os bots scarina efepessoinha. O bot scarina, 


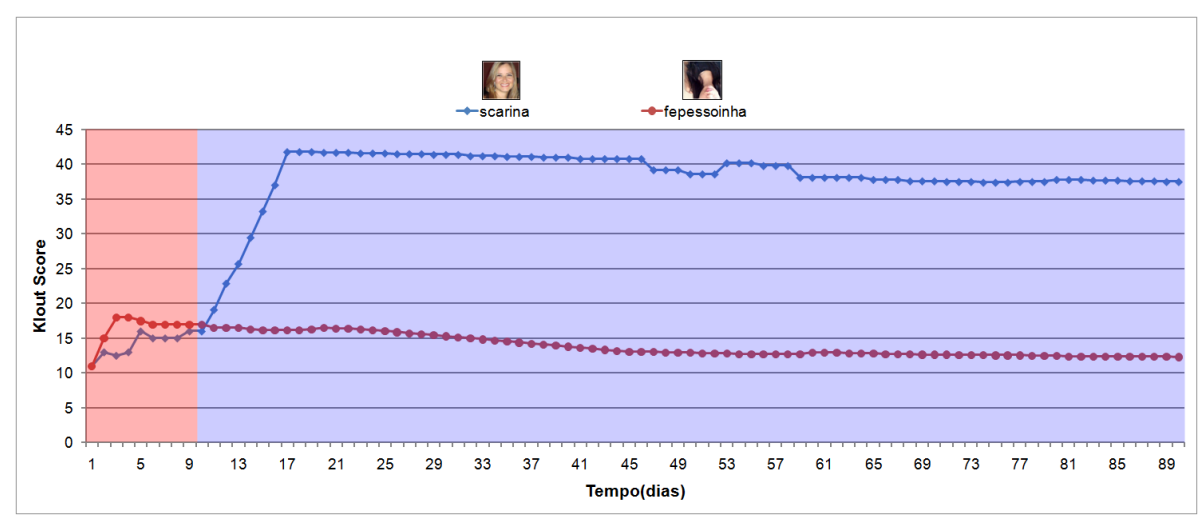

Figura 6. Comparativo do Klout Score entre os bots scarina e fepessoinha

inicia a publicação de tweets a partir do $10^{\circ}$ dia, e no $17^{\circ}$ dia já atinge o valor 41,8 de Klout Score. Observamos no bot scarina, através do Cenário 3, que o bot passa a conquistar novos seguidores mesmo sem seguir outros usuários, apenas pela relevância do conteúdo que posta. O conteúdo que o bot posta acaba sendo de interesse de outros usuários que consequentemente passam a seguir o bot. Com isso, é possível ver que apenas seguir usuários, como realizado no Cenário 1, não é suficiente para atingir um alto grau de influência. Logo, o sistema Klout, considera também as publicações de um usuário, para elevar o nível de influência. Enfim, o bot scarina chegou ao valor máximo de 41,8 no Klout, enquanto o bot fepessoinha alcançou o valor máximo de 18 no Klout.

Além do valor do Klout, o bot scarina conseguiu o valor 86 no Twitalyzer. Uma comparação precisa, dos níveis de influência mais altos, pode ser verificada no gráfico correspondente à Figura 7.

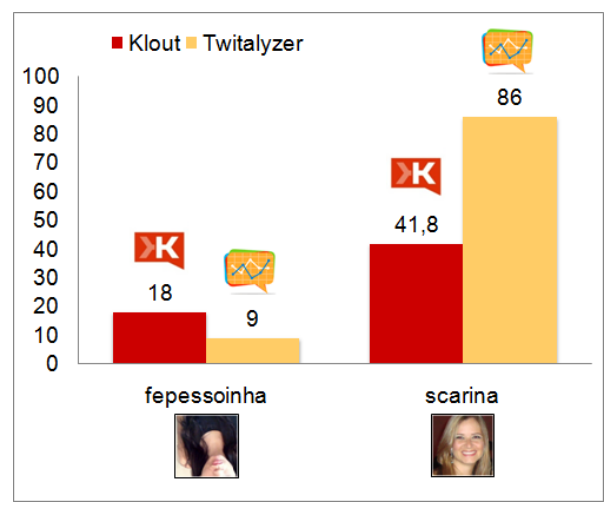

Figura 7. Comparativo Klout e Twitalyzer - fepessoinha x scarina

\subsection{Cenário 4}

Os resultados para o Cenário 4 podem ser vistos claramente no gráfico correspondente à Figura 8. É possível ver grandes vales e picos que correspondem à interrupções na execução do algoritmo de postagem de tweets. Foram duas interrupções. A primeira interrupção se deu no $46^{\circ}$ dia. Mantivemos o algoritmo de postagem de tweets desativado por 6 dias. Reiniciamos o processo completo do bot (coleta/exclusão de usuários e postagem) e mantivemos por mais 6 dias a execução, até realizarmos uma nova interrupção. Reiniciamos 
então apenas o processo de postagem de tweets. Através do gráfico, fica evidente que a não publicação de tweets (com a interrupção do processo) diminuiu bruscamente o grau de influência do bot, baseado no Klout Score.

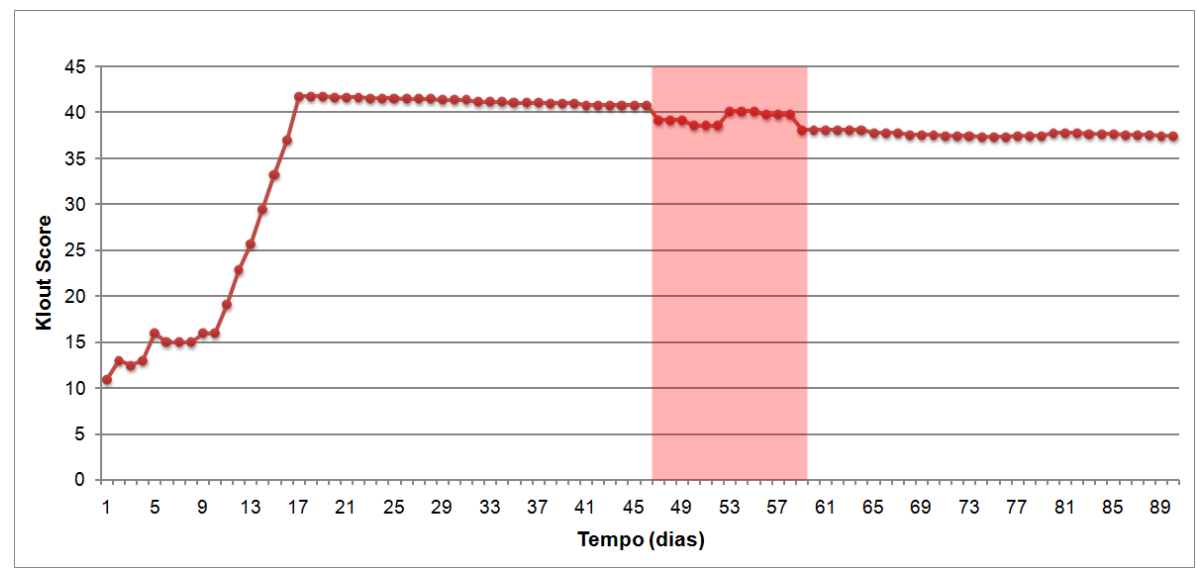

Figura 8. Resultados Klout Score do bot scarina

Conseguimos ver o mesmo comportamento nos gráficos que correspondem aos seguidores, menções e mensagens, principalmente após a primeira interrupção. No gráfico correspondente à Figura 9 podemos ver, a partir do dia 46 que o bot conquista diversos novos seguidores, por executar todo o processo inicial, após a primeira interrupção. Ao final de 90 dias de experimento o bot conquistou o total de 691 seguidores.

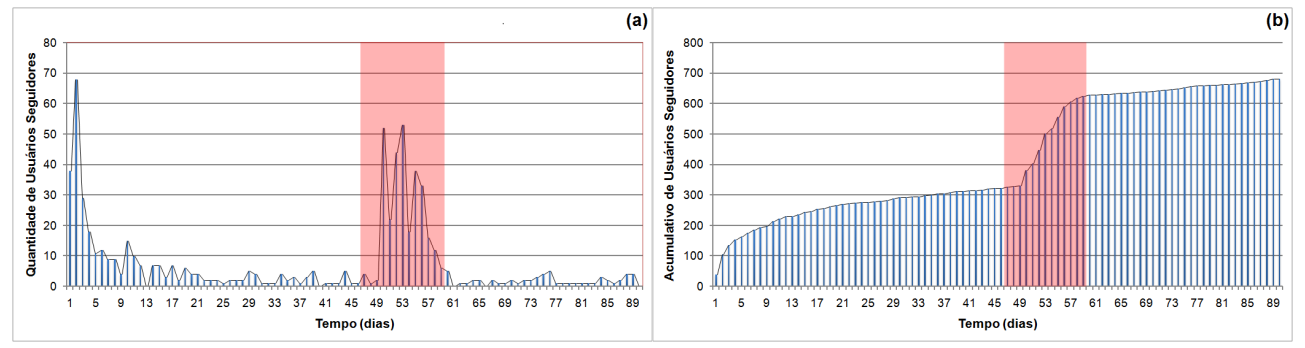

Figura 9. (a) Gráfico de Seguidores do bot scarina (b) Gráfico do acumulativo de seguidores do bot scarina

No gráfico correspondente à Figura 10 também podemos ver, a partir do dia 46 que o bot recebe novas menções e mensagens. Como o processo inicial foi executado novamente, conquistando novos seguidores, a maioria das menções e mensagens foram de questionamentos sobre quem era o bot scarina, e de onde os usuários se conheciam. Ao final de 90 dias de experimento o bot scarina obteve 52 menções e 45 mensagens.

Além dos resultados já apresentados, o bot scarina postou 4997 tweets, durante os 90 dias de experimento. Deste total, tivemos apenas 6 tweets entre os favoritos pelos usuários seguidores. Além disso, obteve ao fim dos 90 dias de experimento o total de 94 retweets e 109 tweets respondidos. 


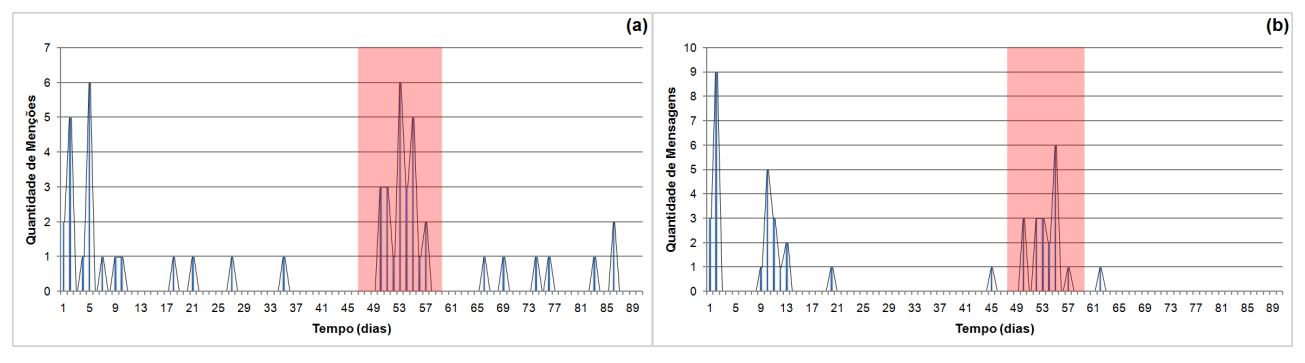

Figura 10. (a) Gráfico de Tweets mencionados do bot scarina (b) Gráfico de mensagens do bot scarina

\subsection{Interações com famosos}

\subsection{Resultados finais}

No final dos 90 dias de experimento, coletamos os valores de Klout e Twitalyzer das contas mais influentes do Twitter e fizemos uma comparação final com os bots fepessoinha e scarina, conforme é mostrado no gráfico correspondente à Figura 11. Entre os mais influentes, estão o humorista brasileiro Rafinha Bastos, o rapper americano Snoop Dogg, o presidente dos Estados Unidos Barack Obama e o apresentador brasileiro Luciano Huck. Podemos ver que mesmo de forma automatizada, os bots alcançaram níveis iguais ou acima, de outras pessoas com alta reputação, nos classificadores de influência Klout e Twitalyzer.

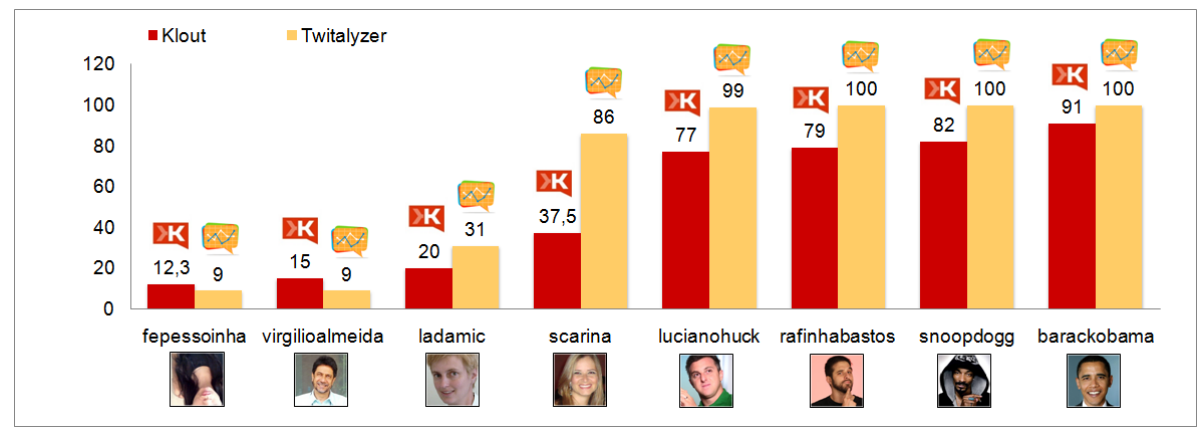

Figura 11. Comparação Klout e Twitalyzer - os mais influentes

\section{Trabalhos Futuros}

Com a apresentação e comparação de algumas estratégias utilizadas por bots na rede social Twitter, este trabalho servirá como base para o estudo e comparação de outras estratégias de influencias nas redes sociais. Um incremento interessante seria utilizar o bot scarina com o dicionário de dados baseado nos trends topics do próprio Twitter, de forma dinâmica. Outra alternativa, seria utilizar estratégias de PLN (Processamento de Linguagem Natural) visando a geração e compreensão automática de línguas humanas naturais, no tratamento dos tweets postados pelos usuários. Com isso, o bot seria capaz de interagir, de forma automática postando conteúdos relacionados ao contexto em que for inserido. Enfim, avaliar mais detalhadamente as melhores estratégias que os bots podem usar para manipular rankings de influencias em redes sociais. 


\section{Conclusões}

Neste artigo criamos robôs simples capazes de interagir, através de contas no Twitter, como se fossem usuários na rede, trocando informações, seguindo e conquistando novos seguidores, durante o período de 90 dias. Constatamos que durante este período, os bots conseguiram resultados significantes em sistemas de classificadores de influência como Klout e Twitalyzer.

Artigos científicos e a mídia em geral têm usado esses sistemas, exageradamente, como padrão para avaliar a influência de um usuário nas redes sociais. Ao decorrer do trabalho, conseguimos constatar que suas métricas são vulneráveis e facilmente manipuláveis quando utilizamos contas automatizadas, conhecidas como bots. Com isso, mostramos que simples robôs no Twitter podem conseguir altos níveis de influência, segundo as métricas do Klout e Twitalyzer, confirmando nossa hipótese. Além disso, verificamos de forma experimental e prática algumas estratégias para os bots. A estratégia de apenas seguir usuários e ser seguido por outros usuários não é o suficiente para um alto grau de influência. Como melhor estratégia, verificamos que o bot também deve interagir postando conteúdos relevantes na rede.

Finalmente, ao fim do experimento, para comprovar a eficácia dos nossos algoritmos, em um curto período, nossos bots conquistaram níveis próximos à níveis de personalidades e pessoas com alta reputação, que são consideradas as mais influentes no Twitter. Com isso, mostramos que processos automáticos com bots podem conquistar altos níveis de influência.

\section{Referências}

[1] Klout. http://www.klout. com, March 2012.

[2] Twitalyzer. http://www.twitalyzer.com/, March 2012.

[3] Purohit H., Ruan Y., Joshi A., Parthasarathy S., and Sheth A. Understanding user-community engagement by multifaceted features: A case study on twitter. In Conference of Social Media Analytics Workshop at World Wide Web (WWW), 2011.

[4] Yan J. L. S. and Kaziunas E. What is a tweet worth?: measuring the value of social media for an academic institution. In Proceedings of the 2012 iConference, iConference '12, pages 565-566, New York, NY, USA, 2012. ACM.

[5] David Leonhardt. A better way to measure twitter influence. The New York Times, 2011.

[6] Cha M., Haddadi H., Benevenuto F., and Gummadi K. P. Measuring User Influence in Twitter: The Million Follower Fallacy. In AAAI ICWSM, May 2010.

[7] Romero D. M., Galuba W., Asur S., and Huberman B. A. Influence and passivity in social media. In $A C M W W W$, pages 113-114, 2011.

[8] Weng J., Ee-Peng Lim, Jiang J., and He Q. Twitterrank: finding topic-sensitive influential twitterers. In ACM WSDM, 2010.

[9] Pal A. and Counts S. Identifying topical authorities in microblogs. In ACM WSDM, pages 45-54, 2011.

[10] Ghosh S., Viswanath B., Kooti F., Sharma N. K., Gautam K., Benevenuto F., Ganguly N., and Gummadi K. Understanding and Combating Link Farming in the Twitter Social Network. In Proceedings of the 21st International World Wide Web Conference (WWW'12), Lyon, France, April 2012.

[11] Boshmaf Y., Muslukhov I., Beznosov K., and Ripeanu M. The socialbot network: When bots socialize for fame and money. In ACSAC'11 Proceedings of the 27th Annual Computer Security Applications Conference, pages 93-102, New York, NY, USA, 2011. 